\title{
Light-Shift Suppression with Novel Variants of Adaptive Ramsey Spectroscopy
}

\author{
M. Shuker, J. W. Pollock, J. Kitching, and E. A. \\ Donley \\ National Institute of Standards and Technology, \\ Boulder, Colorado, 80305, USA \\ and University of Colorado, \\ Boulder, Colorado, 80309-0440, USA \\ V. I. Yudin \\ Novosibirsk State University, \\ ul. Pirogova 2, Novosibirsk, 630090, Russia \\ and Institute of Laser Physics SB RAS, \\ pr. Akademika Lavrent'eva 13/3, Novosibirsk, 630090, \\ Russia \\ and Novosibirsk State Technical University, \\ pr. Karla Marksa 20, Novosibirsk, 630073, Russia
}

\author{
R. Boudot \\ FEMTO-ST, CNRS \\ 26 rue de l'Épitaphe, 25030, Besancon, France \\ and National Institute of Standards and Technology, \\ Boulder, Colorado, 80305, USA
}

\begin{abstract}
We present a brief review of the rapidly growing field of autobalanced Ramsey spectroscopy followed by a detailed discussion and review of two novel techniques that were developed and tested in our laboratory: displaced frequency-jump Ramsey spectroscopy and combined error signal spectroscopy. These two techniques are related to, yet different from autobalanced Ramsey spectroscopy. The use of these techniques in a cold-atom clock based on coherent population trapping has reduced instabilities from variations in light-shift parameters by at least one order of magnitude. In each of these techniques the Ramsey sequence adapts to cancel light shifts and drifts.
\end{abstract}

Keywords—atomic clocks; light shifts; Ramsey spectroscopy

\section{BACKGROUND}

Ramsey's method of separated oscillating fields [1] is a powerful technique that is widely used for the measurement of atomic transition frequencies. With standard Ramsey spectroscopy, two short interrogation pulses are separated by a dark period, with the first pulse generating the atomic coherence and the second pulse probing its phase. Although the atoms spend most of their time in the dark, non-negligible residual sensitivity to light shifts is observed due to shifts that arise from the interrogation pulses. These light shifts cause systematic frequency shifts and instability in optical atomic clocks [2] as well as clocks based on coherent population trapping (CPT), which probe microwave transitions optically [3-5].

Sophisticated interrogation protocols based on Ramsey's method and tailored composite laser pulses have been proposed and demonstrated that can eliminate probe-induced frequency shifts (for a review, see [6]). Key to these techniques is that the light shift magnitude for standard Ramsey spectroscopy depends on the duration of the Ramsey dark period, scaling as $1 / T$. The light shift also depends on several parameters related to the pulses themselves, including the interrogation frequency, phase, and/or pulse length. With a technique called hyperRamsey spectroscopy [7], the pulse parameters can be tuned to form composite pulses that compensate for the light shifts. One can determine the appropriate form of the composite pulses by performing Ramsey frequency measurements with various composite pulses for different values of $T$, and tuning the pulse parameters until the central fringe frequency becomes independent of $T$, at which point light shift vanishes.

Autobalanced Ramsey (ABR) spectroscopy is a technique that takes the removal of light shifts one step further by actively adjusting the composite pulses to stabilize against the shifts [8]. With ABR, two error signals derived from interleaved Ramsey sequences of different dark periods drive two feedback loops, with one feedback loop applied to the clock frequency and one feedback loop used to control a phase correction applied between the pulses. Autobalanced Ramsey spectroscopy was proposed and originally demonstrated in a ytterbium single-ion clock [8], in which a phase correction was applied to the second Ramsey pulse, thus cancelling the light shift. The method was later applied to vapor-cell clocks based on CPT to show a significant improvement in mid-term stability $[9,10]$.

The ABR technique has also been generalized theoretically to show that a variety of secondary variables (or concomitant parameters) in addition to a controlled phase correction can be used for the composite pulses, including external frequencystep compensation and variable second-pulse duration [11]. 


\section{DISPLACED FREQUENCY JUMP RAMSEY}

A new technique called displaced frequency-jump Ramsey spectroscopy (DFJR) was recently demonstrated [12], which is related to but different from ABR in a few important ways. The fringes in Figure 1 illustrate the concept of the DFJR technique.

Like ABR, it uses two interleaved Ramsey sequences with different dark periods and uses two control loops, but it does not use adjusted composite pulses in one of the control loops. Instead, the controlled concomitant parameter, $\alpha$, is a scaling factor for a finely adjusted frequency displacement that is inversely proportional to the dark period and applied to the frequency jumps that probe the sides of the central Ramsey fringe: $\Delta f_{T}=\alpha / T$. Thus, the probe frequency of the local oscillator (LO) modulates about the central fringe in the Ramsey spectrum like usual in a Ramsey-style clock, but with a servoed frequency displacement having a 1 / $T$ dependence determined from interleaved sequences of different dark periods. This displacement is tracked in software, and when it is subtracted from central Ramsey fringe frequency, the result is the unshifted clock frequency, $f_{c}$. The value of the light shift is measured by the scaled frequency displacement $\Delta f_{T}$. The DFJR method has been shown to suppress light shifts in the cold-atom CPT clock developed at NIST [13-15] by more than one order of magnitude [12] to the $1 \times 10^{-12}$ level or lower in our experiment.

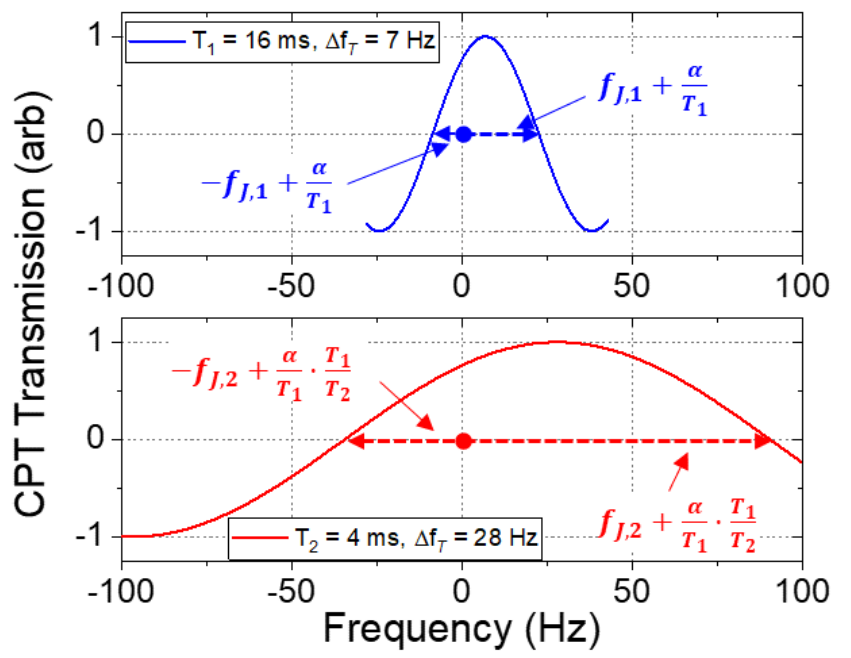

Fig. 1. Simulated light-shifted central Ramsey fringes for two different dark periods illustrating the concept of the DFJR technique. In the top figure, the Ramsey period was $16 \mathrm{~ms}$, giving a fringe width at half maximum of $31.25 \mathrm{~Hz}$. The light shift, $\Delta f_{T 1}=\alpha / T_{1}$, was assumed to be $7 \mathrm{~Hz}$ (made large for illustrative purposes). In the bottom figure, the Ramsey period was $4 \mathrm{~ms}$, the fringe width was $125 \mathrm{~Hz}$, and since the Ramsey period was four times shorter than in the top figure, the light shift, $28 \mathrm{~Hz}$, was four times larger. The frequency jumps for the servo modulation, $f_{J, 1(2)}=1 /\left(4 \cdot T_{1(2)}\right)$, are different for the two dark periods and are offset with the second control loop by the frequency displacement $\Delta f_{T} . \Delta f_{T}$ is found along with the clock frequency $f_{\mathrm{c}}$ by interleaving Ramsey sequences with two different dark periods. In effect, the short Ramsey sequences steer the frequency displacement $\Delta f_{T}=\alpha / T$ to compensate for the light shift and the long Ramsey sequences steer the clock frequency $f_{\mathrm{c}}$ to the unshifted frequency $f_{0}$.

There are a few technical factors that make DFJR attractive in practice. The control parameters, $\alpha$ and $f_{c}$, are controlled with a single physical variable - the LO frequency. Modulation of the LO phase is not needed, which allows for simple implementation without systematics that can arise from modulating the oscillator phase. Also, unlike one variant of generalized ABR [11] where frequency steps are applied during the Ramsey pulses, here the LO frequency remains constant during the Ramsey cycle, and no rapid control of the LO frequency is required.

\section{COMBINED ERROR SignAL RAMSEY}

It has also been shown theoretically [16] and experimentally [17] that probe-induced light shifts can be removed by combining two error signals into a single error signal with a method called combined error signal Ramsey spectroscopy (CES) [16]. The error signal steers the clock frequency to its unshifted value without requiring a concomitant parameter. Like before, the two error signals are generated with alternating Ramsey sequences. To combine the signals with the appropriate weights, the error signals are normalized by a factor that equalizes the Ramsey fringe amplitude and then subtracted. The method is simple since there is a single control loop with the clock frequency as a single control parameter.

A challenge with implementing this method is to determine the appropriate normalization factors, but there is a natural way to do this with NIST's cold-atom CPT clock, in which the main decay mechanism arises from atoms escaping the probe volume after they are released from the optical molasses. So, the normalization process in this case can easily be accomplished by inserting a delay before the shorter Ramsey pulse in the sequence such that the probe pulses (the $2^{\text {nd }}$ Ramsey pulses) occur after the same release period from the molasses for the long and short Ramsey sequences. The CES method has also been shown to suppress light shifts by at least one order of magnitude over traditional Ramsey-CPT spectroscopy [17].

\section{OUTLOOK}

So far, our work has focused on quantifying light-shift suppression in our cold-atom clock at NIST. An ongoing important focus of research will be to measure how much the long-term stability of CPT clocks can be improved by these techniques, and the apparatus is currently undergoing an upgrade to be able to perform these measurements.

Autobalanced Ramsey and other adaptive spectroscopy methods are a new field with many interesting potential applications. In principle, it should be possible to remove systematics other than light shifts when there is a model that closely predicts the scaling of the shift versus $T$.

\section{ACKNOWLEDGMENT}

The authors thank V. Maurice, L. Stern, and M. Lombardi for helpful suggestions on this manuscript. V. Maurice is also gratefully acknowledged for writing the software used to perform the experiments. V. I. Yudin was supported by the Russian Science Foundation (No. 16-12-10147), Ministry of Education and Science of the Russian Federation (No. 3.1326.2017/4.6), and Russian Foundation for Basic Research (No. 17-02-00570). A. V. Taichenachev was supported from Russian Science Foundation (No. 16-12-00054) and Russian Foundation for Basic Research (No. 18-02-00822). R. Boudot was supported by the NIST Guest Researcher Fellowship and Délégation Générale de l’Armement (DGA). 


\section{REFERENCES}

N. F. Ramsey, "A Molecular Beam Resonance Method with Separated Oscillating Fields," Physical Review 78, 695-699, (1950).

[2] A. D. Ludlow, M. M. Boyd, J. Ye, E. Peik, and P. O. Schmidt, "Optical atomic clocks," Rev. Mod. Phys. 87, 637-701, 2015.

[3] E. Arimondo, "Coherent population trapping in laser spectroscopy," Progress in Optics 35, 257-354, 1996.

[4] J. Vanier, "Atomic clocks based on coherent population trapping: a review," App. Phys. B 81, 421-442, 2005.

[5] V. Shah and J. Kitching, "Advances in Coherent Population Trapping Atomic Clocks," Advances in Atomic, Molecular, and Optical Physics 59, 21-74, 2010.

[6] T. Zanon-Willette et al., "Composite laser-pulses spectroscopy for high-accuracy optical clocks: a review of recent progress and perspectives," Rep. Prog. Phys. 81, 094401, 2018.

[7] V. I. Yudin et al., "Hyper-Ramsey spectroscopy of optical clock transitions," Phys. Rev. A 82, 011804(R), 2010.

[8] C. Sanner, N. Huntemann, R. Lange, C. Tamm, and E. Peik, "Autobalanced Ramsey Spectroscopy," Phys. Rev. Lett. 120, 053602, 2018.

[9] M. Abdel Hafiz et al., "Toward a High-Stability Coherent Population Trapping Cs Vapor-Cell Atomic Clock Using Autobalanced Ramsey Spectroscopy," Phys. Rev. Applied 9, 064002, 2018

[10] M. Abdel Hafiz, G. Coget, M. Petersen, C. E. Calosso, S. Guérandel, E. de Clercq, and R. Boudot, "Symmetric autobalanced Ramsey interrogation for high-performance coherent-population-trapping vapor-cell atomic clock," App. Phys. Lett. 112, 244102, 2018.

[11] V. I. Yudin et al., "Generalized Autobalanced Ramsey Spectroscopy of Clock Transitions," Phys. Rev. Applied 9, 054034, 2018.

[12] M. Shuker, J. W. Pollock, R. Boudot, V. I. Yudin, A. V. Taichenachev, J. Kitching, and E. A. Donley, "Ramsey Spectroscopy with Displaced Frequency Jumps," Phys. Rev. Lett. 122, 113601, 2019.

[13] F. X. Esnault, E. Blanshan, E. N. Ivanov, R. E. Scholten, J. Kitching, and E. A. Donley, "Cold-atom double- $\Lambda$ coherent population trapping clock," Phys. Rev. A 88, 4, 042120, 2013.

[14] X. Liu, E. Ivanov, V. I. Yudin, J. Kitching, and E. A. Donley, "LowDrift Coherent Population Trapping Clock Based on Laser-Cooled Atoms and High-Coherence Excitation Fields," Phys. Rev. Applied 8, 054001, 2017.

[15] J. W. Pollock et al., "ac Stark shifts of dark resonances probed with Ramsey spectroscopy," Phys. Rev. A 98, 053424, 2018.

[16] V. I. Yudin et al., "Combined error signal in Ramsey spectroscopy of clock transitions," New J. of Physics 20, 123016, 2018.

[17] M. Shuker, J. W. Pollock, R. Boudot, V. I. Yudin, A. V. Taichenachev, J. Kitching, and E. A. Donley, "Reduction of light shifts in Ramsey spectroscopy with a combined error signal," App. Phys. Lett. 114, 141106, 2019 